\title{
Approaches to a Successful Myringoplasty - Which Factors Make a Myringoplasty Difficult?
}

\author{
https://doi.org/10.47210/bjohns.2021.v29i1.462
}

Wendy Smith, ${ }^{1}$ Swagatam Banerjee ${ }^{2}$

\section{Introduction}

\section{ABSTRACT}

Myringoplasty has often been viewed as a "basic" ENT procedure. This paper will discuss the factors that can make a myringoplasty more challenging and suggest some techniques to overcome these.

Affecting Factors

Patient factors

The age of the patient, their habitus and co-existing co-morbidities are usually the factors affecting the choice and outcome of the procedure.

The ear

Anatomical variations, the nature of the pathology itself and the condition of the remnant tympanic membrane and the middle ear are important factors for consideration.

Other factors

Equipment availability, expertise of the surgical team and anaesthetic support can also influence the surgical procedure.

Surgical Procedure

The actual procedure can be done in a variety of ways including but not limited to different approaches, different techniques and using different equipment like microscopes, endoscopes, LASER, etc.

\section{Results and Complications}

The primary author reported a success rate of about $95 \%$ in her series. Complications of myringoplasty include myringitis, residual perforations, otitis externa and epithelial pearl formation.

Kevwords

myringoplasty; Otologic Surgical Procedures; Ear

$\mathrm{C}$ hronic otitis media can result in a tympanic membrane perforation that fails to heal spontaneously. Philip Robinson et al published a study performed over 20 years in Bristol in the UK

1 - Department of ENT and Head Neck Surgery, Kettering General Hospital NHS Foundation Trust, Kettering, United Kingdom

2 - Department of ENT and Head Neck Surgery, University Hospitals Coventry and Warwickshire, Coventry, United Kingdom

\section{Corresponding author:}

Swagatam Banerjee

email: swagatam.banerjee1@nhs.net where they observed the natural history of 2865 episodes of perforation to study the rate of natural closure. ${ }^{1}$ After two and a half years observation a staggering $90 \%$ of children under 7 had spontaneous closure of the perforation and even those aged 7 to 12 years had a $75 \%$ spontaneous closure rate. The study recommended that in children there was little point of waiting more than 2 and a half years, the median time for closure overall being 13 months but this is age dependent.

By closing the defect, the patient can return to life without the need for water precautions when showering, washing hair or swimming. Although myringoplasty is not "sold" to improve hearing, closure of posterior or large perforations can improve the conductive hearing 
loss. Philips et al analysed 495 primary myringoplasties from data entered into the Common Otology Database and found an overall improvement in the hearing of those that were successful to be $9.14 \mathrm{dBHL} \pm 10.62 \mathrm{dBHL}{ }^{2}$ Perhaps the only contra-indication to myringoplasty is a dry tympanic perforation in the only hearing ear or in patients with significant co-morbidities.

Myringoplasty has often been viewed as a "basic" ENT procedure and is allocated to junior doctors with little training or supervision resulting in poor success rates. To successfully perform a myringoplasty surgeons need to master many of the principles of good tissue handling and exposure techniques applied in more major ear surgeries. This paper will discuss the factors that can make a myringoplasty more challenging and suggest some techniques to overcome these.

So, can one technique be used for all myringoplasties? If not how do we decide what technique should be used for each patient? As surgeons we make hundreds of decisions subconsciously based on our experience and this may only become apparent when supervising our trainees when we are surprised by their decisions. Factors that need to be considered include those related to the patient, the ear, the surgeon and anaesthetic technique, equipment available and more recently the impact of the Covid-19 pandemic.

\section{Patient factors}

The age of the patient: Patients who are very young or old will present different challenges. Older patients may have very limited neck movement so if it is difficult to perform microsuction in the clinic with the patient on a couch, patient positioning in the operating theatre is likely to be suboptimal.

The minimum age the patient should be before a myringoplasty is offered has been debated. Vrabec performed a meta-analysis which suggested that myringoplasty success improves with age and so it may be better if the child is more than 6 years old however there are very few studies with children less than 6 years ${ }^{3}$. Vrabec also found no correlation of success based on the presence of contralateral ear disease. It is reasonable to consider a myringoplasty in children who despite adequate water precautions still have recurrent ear infections and if hearing loss is present closure of posterior or subtotal perforations may in itself improve the hearing or at least enable the use of a conventional hearing aid without increasing the risk of infection. Hardman et al found in a meta-analysis that adenoidal hypertrophy and surgeon experience can influence the outcome. ${ }^{4}$

In 1983 Singh and Raine recommended surgery to be performed in children over 12 years of age whereas the more recent studies found age did not affect the success of myringoplasty. ${ }^{5}$ Recently more surgeons are performing myringoplasty in younger children the author has successfully operated in those over 6 years old.

It is difficult to determine the success rates of paediatric myringoplasty since a review of the literature identifies studies, all of which have been retrospective, with rates varying from 35 to $93 \%$ with different success criteria. Comparison of the studies is complicated due to inclusions of perforations due to different aetiology which may also influence the success rates. An intact TM appears to be achievable in $60-93 \%$ however if success includes an improvement in hearing with no middle ear disease the success rate falls to $50-70 \%$. In addition, reperforation, glue ear formation and atelectasis can occur in a fifth of patients.

The patient's habitus: Some patients have short, fat necks and large chest and shoulders. This impacts firstly on access, where it may be difficult to use instruments in the correct position and secondly with a raised BMI, there is an increase in the basal metabolic rate as well as other anaesthetic considerations. In these patients, access can be improved by gently pulling down on the shoulder and rotating the table. Suboptimal positioning of the patient can result in a strain on the surgeon's neck and arm especially if the procedure is a longer one.

Other co-morbidities that can affect the surgical outcome: Patients who have had cleft palate repair surgery still have reduced eustachian tube function which will have implications for the success of a myringoplasty. Patients with syndromes such as trisomy 


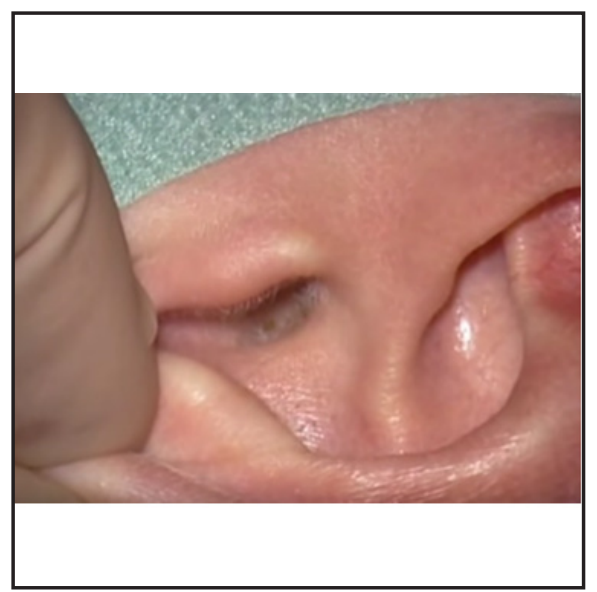

Fig. 1a. A narrow ear canal

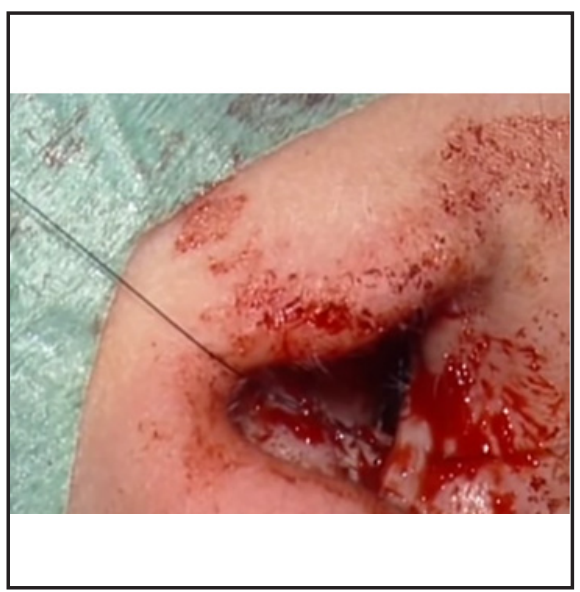

Fig. 1b. Flap shift technique soft tissue meatoplasty

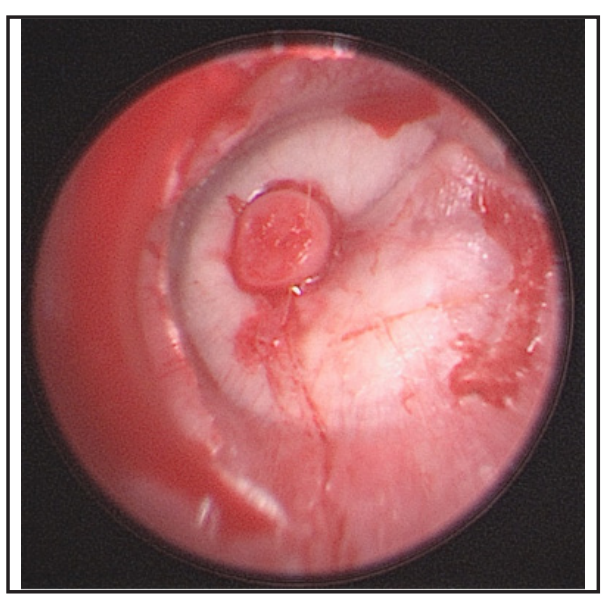

Fig. 2. Butterfly cartilage graft technique
21 may have congenital narrowing of the ear canals as well as eustachian tube dysfunction. Conditions such as scleroderma may present with additional challenges since their tissue quality is poor, not only making lifting of the tympanomeatal flap more difficult, but also affecting the quality of the graft. Patients on anticoagulants are more likely to bleed making a bloodless field more of a challenge to achieve. Patients who are diabetic or are immunocompromised may have higher rate of post-operative infections and prophylactic antibiotics could be advisable.

\section{The ear}

Co-existing ear pathologies: The anatomical sequalae of chronic otitis media varies from a simple central perforation seen after an acute otitis media, trauma, removal of a ventilation tube or be complicated with other conditions such as erosion of the ossicular chain, formation of tympanosclerosis, epithelialization of the middle ear and everted mucosa. These pathologies can affect the surgical procedure of myringoplasty. The middle ear must be carefully inspected to determine the appropriate surgical option not just on the size and position of the perforation but on such complicating pathologies.

The ear canal: Good visualisation is essential and so access via the ear canal must be optimised. Ear surgery in patients with narrow rather than wide ear canals will obviously be more challenging to perform. Although there is a natural tendency to limit the amount of local anaesthetic and adrenaline infiltration to reduce narrowing the ear canal further, this is a mistake and can result in more bleeding. Instead a Lempert endaural speculum can be inserted after injection to gently dilate the ear canal and massage excess infiltration from the skin of the canal. The ear canal be further enlarged after the tympanomeatal flap has been raised using a curette. If the ear canal is very narrow, a soft and/ or a bony canaloplasty may be required. Depending on the soft tissue meatoplasty technique employed, (the author prefers a "flap shift" technique as shown in Fig. $1 \mathrm{a}$ and $\mathrm{b})^{6}$ a staged procedure or an endoscopic butterfly cartilage graft technique to repair the perforation may need to be used as shown in Fig. 2.?

The presence of a prominent anterior canal wall may prevent visualisation of the perforation especially when using a microscope. To close a perforation successfully without complications, the grafting material and meatal skin flaps need to be positioned correctly so a bony canaloplasty may be necessary. The author prefers to use a $2 \mathrm{~mm}$ diamond burr and with a size 18 fine end on a Zollner sucker, the latter is used to retract the tympanomeatal flap that has been raised and reflected superiorly like a bucket handle. Irrigation is not used whilst drilling since this seems to attract the tympanomeatal skin to the drill. Some otologists use 


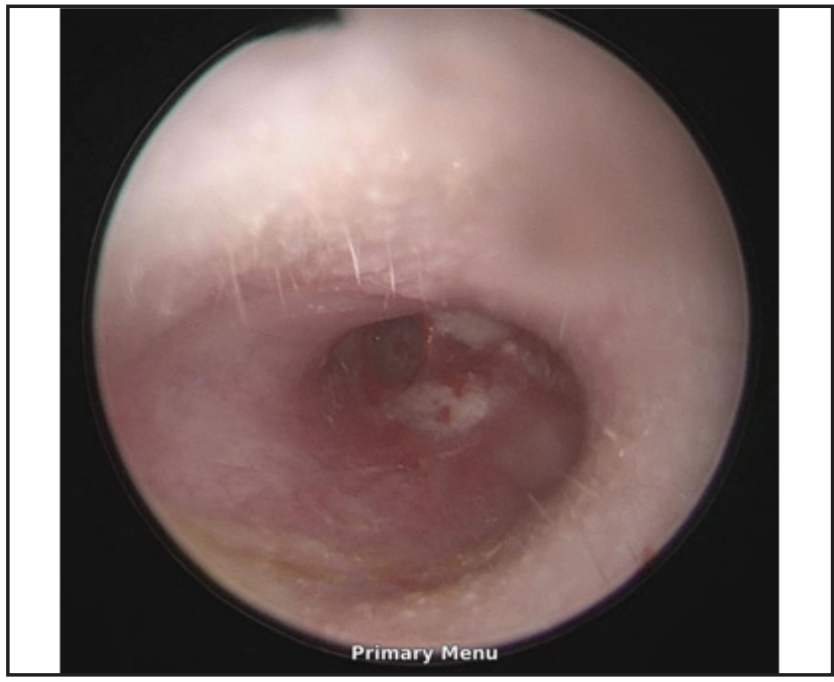

Fig. 3a. Endoscopic view of perforation

foil or silastic sheeting to protect the ear canal skin.

Another way to improve visualisation is using the endoscope. In Fig. 3a the anterior border of the perforation cannot be seen, however just by dropping the hand holding the endoscope, the view is improved as seen in Fig. 3b. With experience, the endoscopic approach can be used to repair the more difficult anterior, subtotal and total perforations.

Sometimes there are co-existing lesions in the ear canal. A patient with a subtotal perforation had a fatty

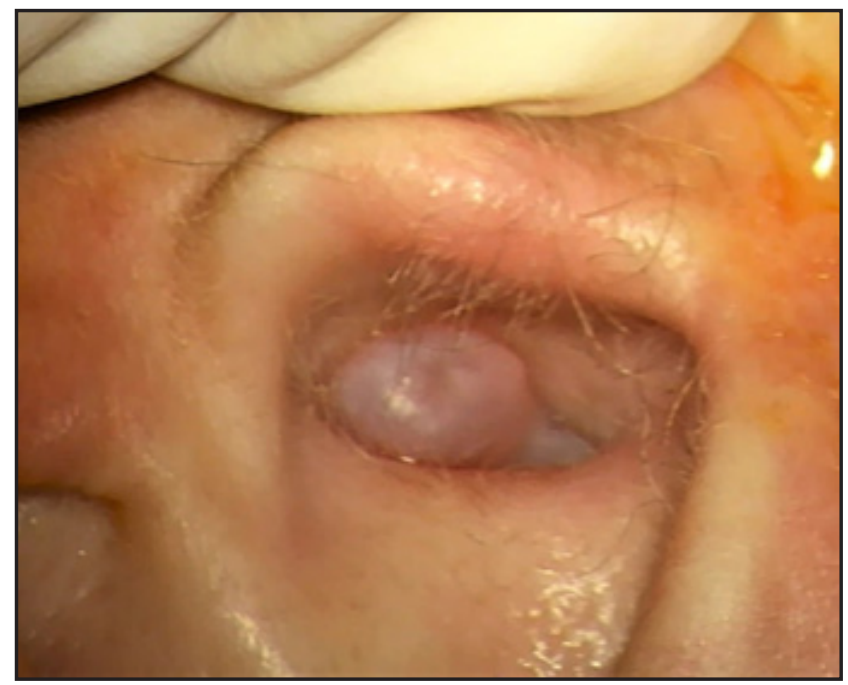

Fig. 4a. Lesion in ear canal

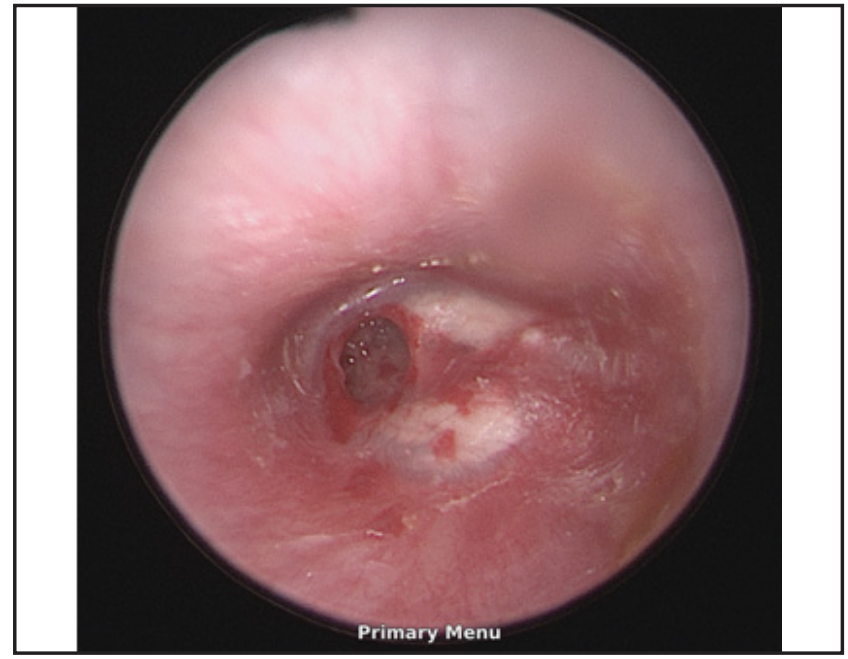

Fig. 3b. improved by lowering hand with the endoscope.

lesion in the ear canal (Fig. 4a) which was removed (Fig. $4 \mathrm{~b})$ before repairing the tympanic membrane during the same operation. Other lesions such as exostosis and osteomas may be encountered and need to be removed prior to performing the myringoplasty, if necessary, as a staged procedure.

Another challenge to the otologist is patients with active otitis externa in the presence of a perforated tympanic membrane. Myringoplasty should only be attempted after treating the otitis externa using

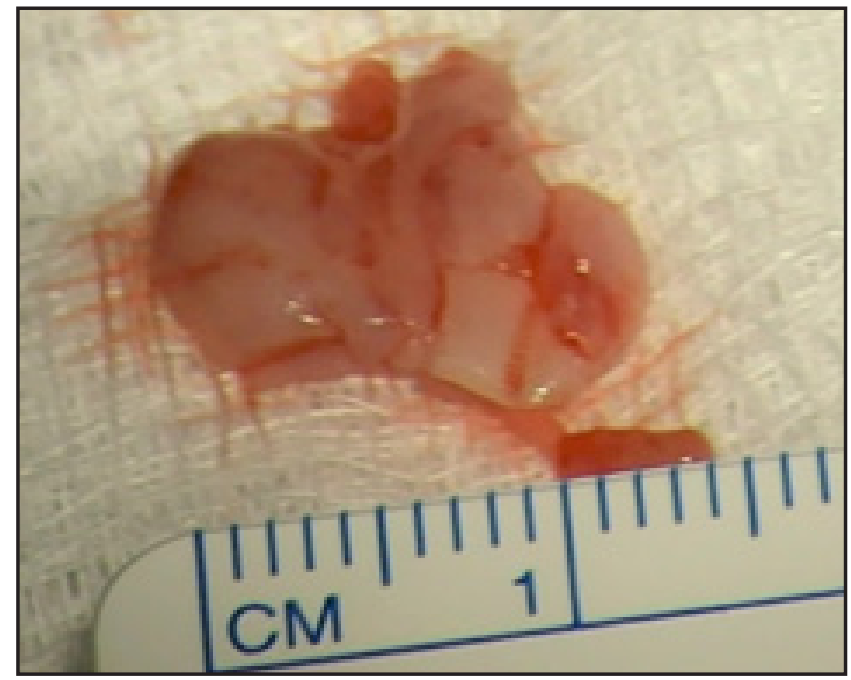

Fig. 4b. A "fatty lesion" was excised 


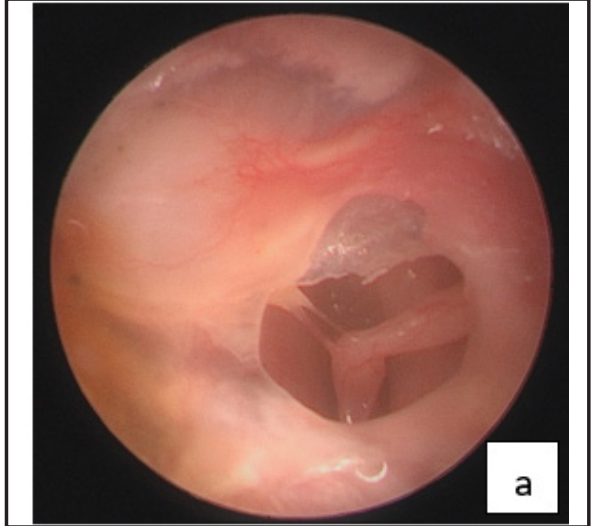

Fig. 5a. A posterior perforation in a left ear with the incudostapedial joint seen along with some adhesions

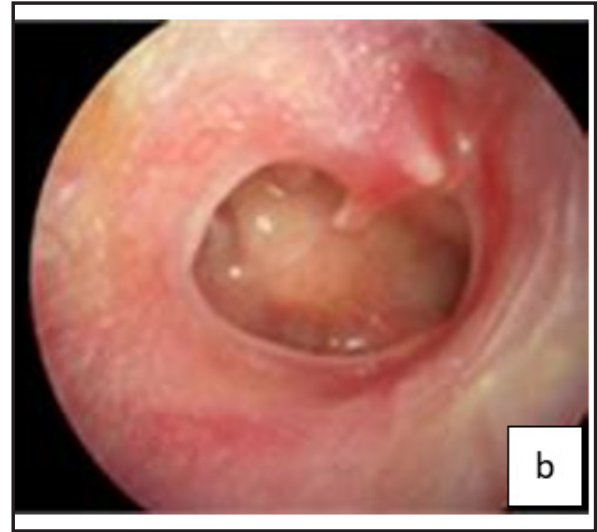

Fig. 5b. The subtotal perforation is complicated further with a medialised malleus

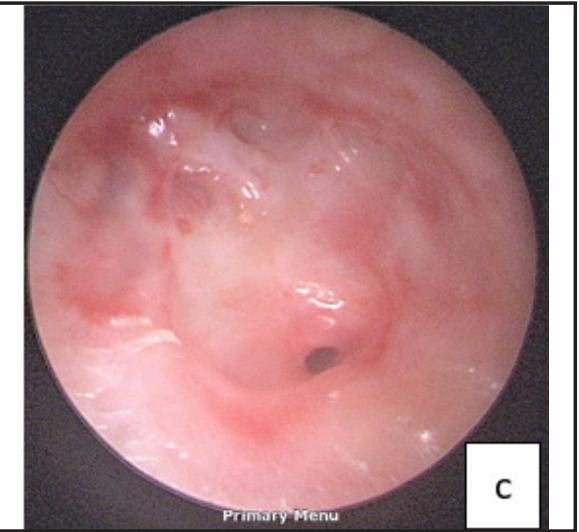

Fig. 5c. A small posterior perforation with perhaps a degree of myringitis appropriate topical drops (according to swab sensitivities) and water precautions.

The perforation itself and the status of the remaining tympanic membrane: Figures $5 \mathrm{a}, \mathrm{b}$ and $\mathrm{c}$ show 3 different perforations varying in size and position each with their own challenges.

The size and site of the perforation needs to be considered. Anterior perforations are more difficult to

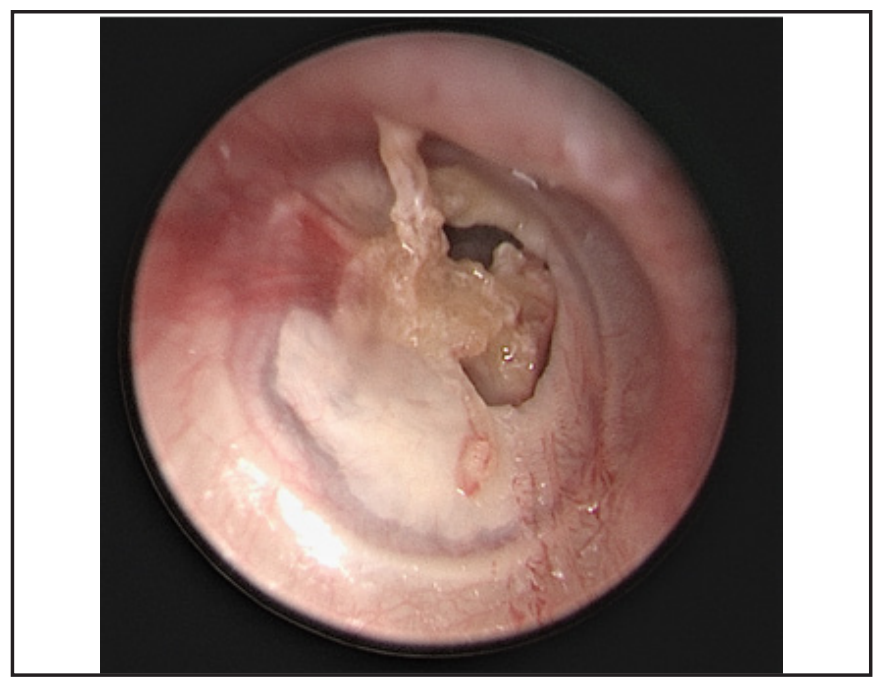

Fig. 6. Squamous epithelium migrating into the middle ear access, and the blood supply is poorer and this affects whether the myringoplasty is likely to be successful. Singh et al had a 34\% success for anterior perforation but $91 \%$ and $100 \%$ for inferior and posterior perforations, respectively. 8 The size of the perforation seems to affect the success rate for some but not others. Lee et al had a success rate of $74 \%$ for small perforations but only $56 \%$ for larger ones whereas Yung did not find size to be a factor. ${ }^{9,10}$

Inflammatory episodes may induce tympanosclerosis

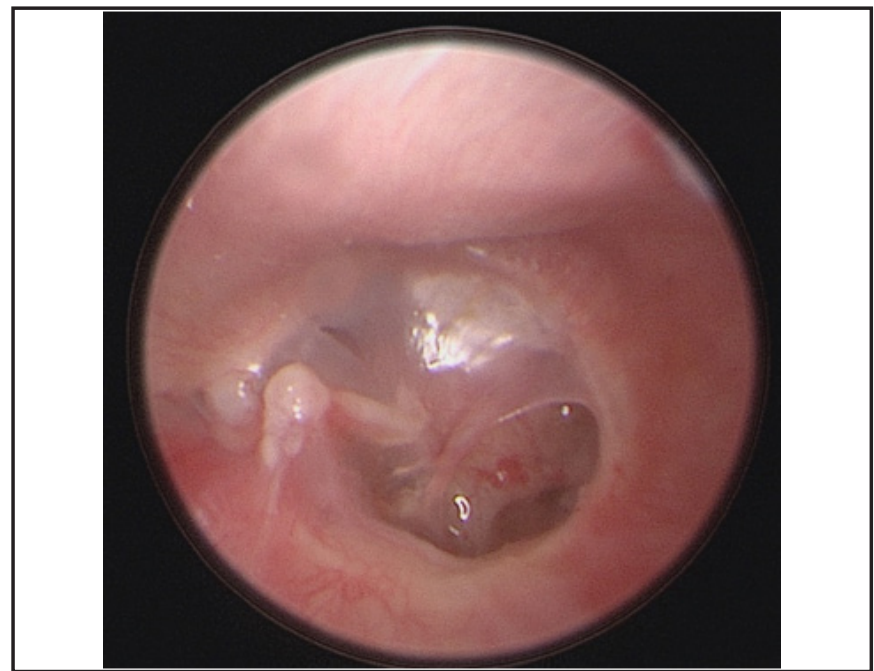

Fig.7. Posterior perforation complicated with medialisation of the handle of malleus and tympanic membrane adherent to promontory 


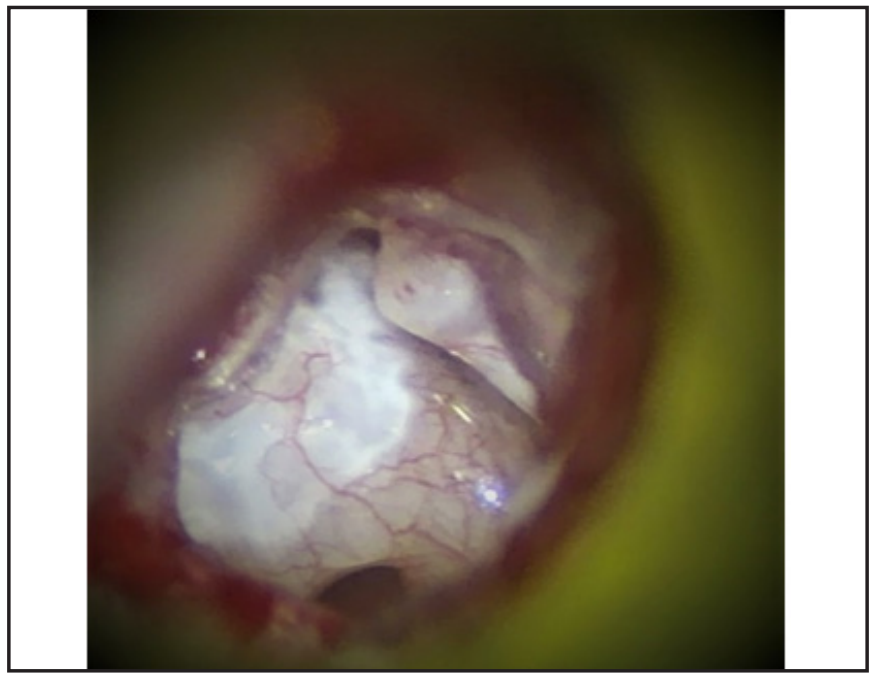

Fig. 8. Tympanic membrane adherent to incudostapedial joint

which histologically is characterized by devascularization and hyalinization of the of the middle ear. The author believes that removal of the tympanosclerotic plaques improves the success rates for myringoplasty perhaps by improving the blood supply to the graft. A tympanosclerotic plaque can be removed from the medial surface of the tympanic membrane without disturbing the epithelial layer or by total removal of the involved tympanic membrane.

Squamous epithelium on the lateral tympanic membrane may advance into the middle ear forming a cholesteatoma (Fig. 6). Sometimes the mucosal layer of the tympanic cavity can advance over the edge of the perforation towards the epidermal layer replacing the squamous epithelium.

The position of the handle of the malleus can make surgery more challenging. In Fig. 7, there is a posterior perforation, the superior margin of which is adherent to the promontory and the malleus handle is medialised. The pre-operative audiogram is an important consideration. If despite the malleus being medialised, the hearing is $30 \mathrm{~dB}$ HL efforts should be made to keep the ossicular chain intact. If a conductive hearing loss of more than $30 \mathrm{~dB}$ is present, then disarticulation of incudo-stapedial joint followed by gently lateralising the malleus enables better placement of the graft. An ossiculoplasty could be performed if necessary.

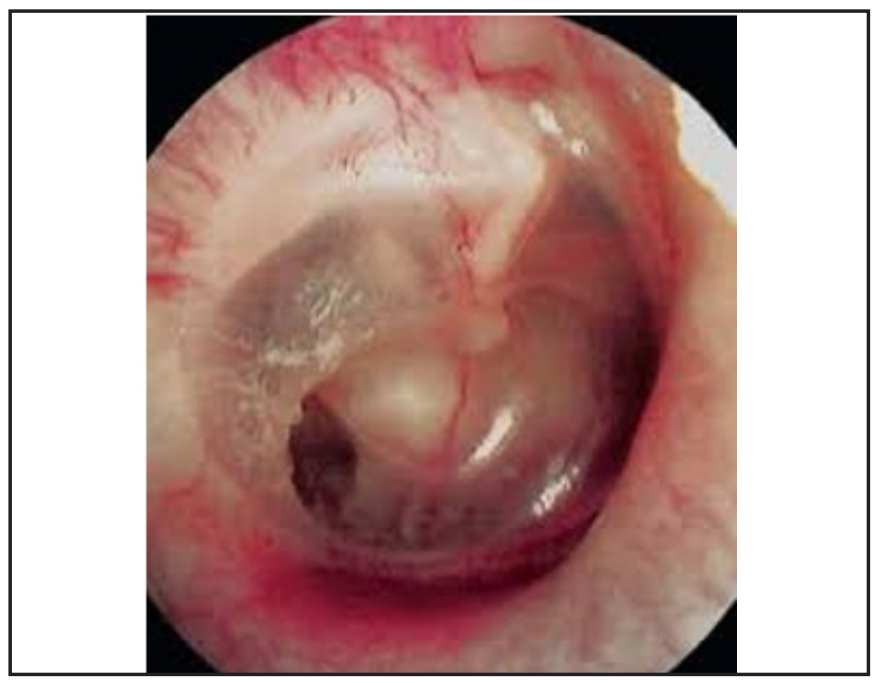

Fig. 9. Tympanic membrane adherent to the promontory.

Where the graft cannot be place using an underlay technique with graft under the malleus- (there is tendency for the malleus to pull the graft from the anterior and posterior malleus ligaments), the malleus handle can be cleaned and a hole or large cut in the in the fascia made so the manubrium can be exteriorised.

Unfortunately, the tympanic membrane can retract onto the incudo-stapedial joint even with a perforation present to ventilate the middle ear as seen in Fig. 8. Experience and patience is required to lift the often very thin tympanic membrane off the ossicular chain without causing a traumatic hearing loss or leaving an epithelial layer from which cholesteatoma can develop.

In Fig. 9 the tympanic membrane is adherent to the promontory but careful elevation of this thin tympanic membrane is necessary to enable graft placement.

The status of the middle ear: Chronic inflammation often causes erosion and/or fixation of the ossicular chain which may be surrounded by hard fibrous scar tissue. This can lead to difficulty raising the tympanic membrane and erosion of the incus and the possible benefits of an ossiculoplasty may need to be considered, taking into account the health status of middle ear. The presence of granulations in the middle ear also leads to more bleeding which can make the surgery more challenging. 


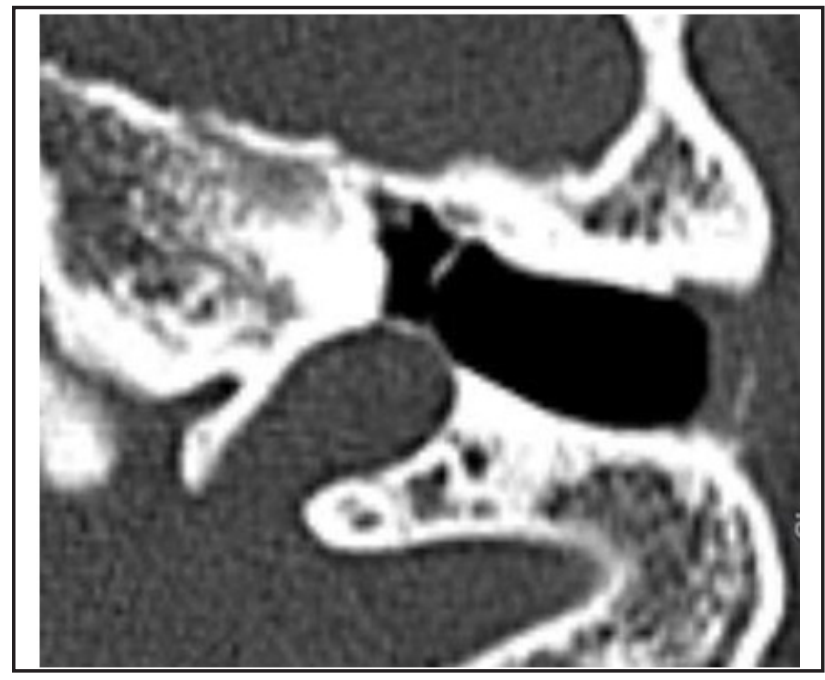

Fig. 10 a. CT scan showing a high jugular bulb

Some patients with tympanic membrane perforations seem to have a continuous discharge from the ear despite attempts to clear it pre-operatively. Tawab et al found that an additional mastoidectomy performed in ears with chronic otitis media without cholesteatoma gave no statistically significant benefit over a simple myringoplasty with regards to graft success rate, discharge and hearing results. ${ }^{11} \mathrm{CT}$ and MRI scans may be required to exclude an underlying cholesteatoma.

Does co-existing cholesteatoma affect the success rates of myringoplasty? Yoon et al did not find the presence of cholesteatoma or in fact presence of discharge in the ear to impact on success rates of myringoplasty. ${ }^{12}$ The author included all pars tensa perforations with and without cholesteatoma present on the common otology database and found no difference in the myringoplasty success rate of over $95 \%$ in over 400 cases. It is important to ensure all cholesteatoma is removed from the undersurface of the tympanic membrane. Sometimes it is best to excise the affected tympanic membrane and the need for follow-up non-EPI DWI MRI scan or second look surgery may be required.

A high dehiscent jugular bulb: Figures $10 \mathrm{a}$ and $\mathrm{b}$ are the CT scans of a male who had a successful combined approach tympanoplasty on the right ear and wanted a myringoplasty so he could finally go

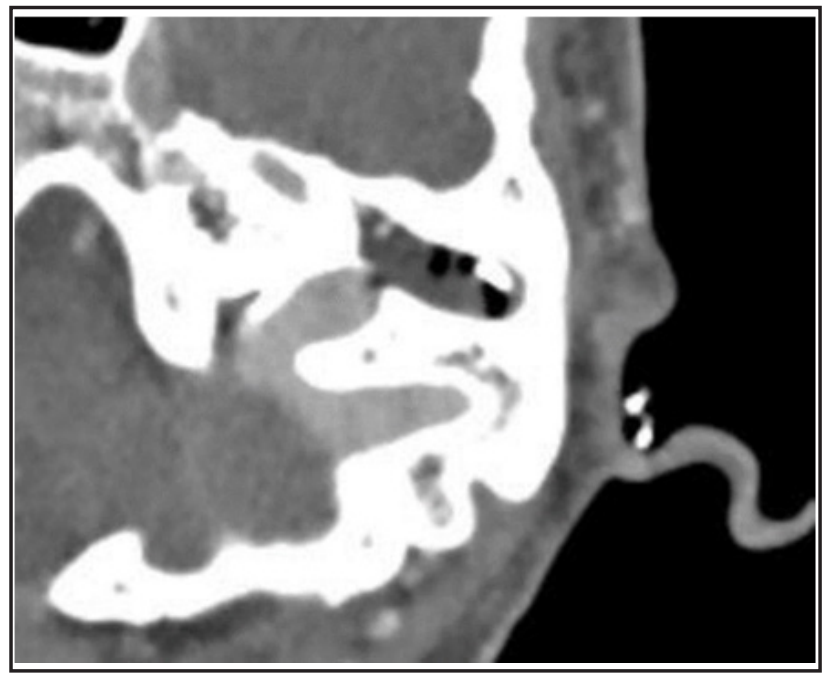

Fig. 10 b. with contrast following surgery

swimming. Unfortunately the scans were not reviewed prior to the myringoplasty and during elevation of the tympanomeatal flap, heavy bleeding was encountered from the exposed high jugular bulb.

So how do you deal with the high dehiscent jugular bulb? If known pre-operatively by reviewing any CT scans you could consider a technique not raising the tympanomeatal flap. After freshening the edge of the perforation, the middle ear below the perforation can be filled with gelfoam/spongostan and then fat or fascia can be tucked under the edge of the perforation to close it. This has been done on 3 occasions having stopped the bleeding using ribbon gauze soaked in adrenaline to pack the ear canal. After 10 minutes pieces the packing is carefully removed from the ear canal and pieces of surgicel inserted in the hypotympanum to stop the bleeding so the operation could be completed.

\section{The myringoplasty technique}

The graft material: Several different biological and non-biological graft materials are used by surgeons. B Nicholas and R O'Reilly reviewed the literature in 2010 and found no significant difference in hearing outcome but cartilage may have better closure rates in the paediatric population. ${ }^{13}$ Fat plug myringoplasty technique has largely been employed for small central 


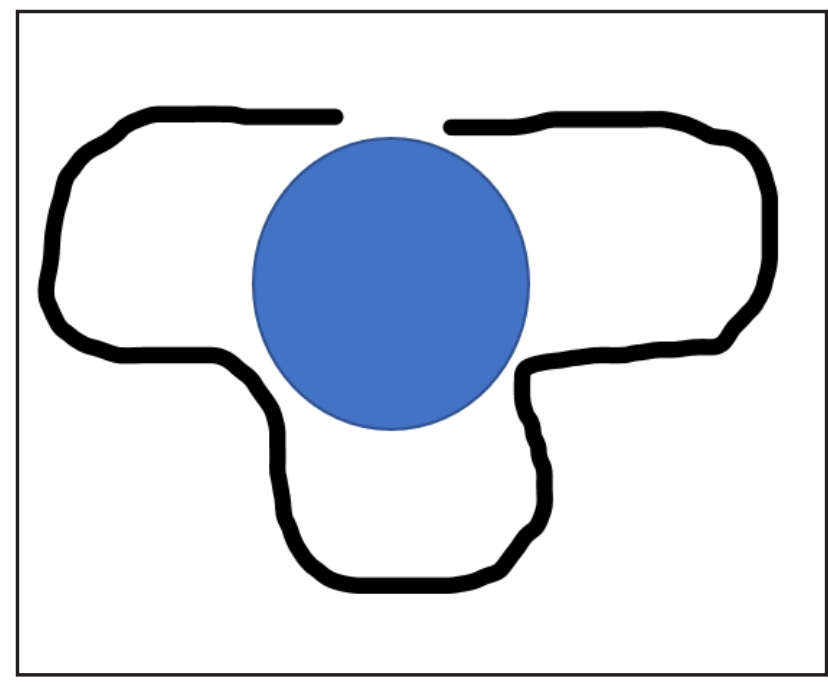

Fig. 11a. Temporalis fascia with 3 "outpouches"

posterior or inferior perforations. Lee et al in 2018 carried out a systematic review and found that fat plug myringoplasty success rates were indeed inferior to those using fascia and perichondrium especially in larger and anterior perforations. ${ }^{14}$ Others have found that the hearing outcomes are worse for those with larger perforations.

The author favours the use temporalis fascia if a postaural approach has been used but tragal cartilage in permeatal surgeries whether using the endoscope or microscope. Biodesign has been chosen when previous surgery had utilised the available fascia and cartilage. Biodesign is natural extracellular matrix derived from porcine small intestinal submucosa that acts as a scaffold for the body's cells to infiltrate and remodel into vascularised tissue. Closure rates are reported to be comparable with temporalis fascia, ranging from $83 \%$ $-100 \%$ across published literature. ${ }^{15-17}$

When harvesting tragal cartilage, the skin incision is made a few $\mathrm{mm}$ below the rim of the tragus and $3-4 \mathrm{~mm}$ of cartilage is kept laterally which improves the cosmesis but should the patient require a conventional hearing aid in the future, this retained cartilage improves the fitting of the aid. Sometimes only perichondrium is required. When cartilage on perichondrium is used, a rim of perichondrium is fashioned which then lies on the bony annulus. The amount of cartilage required depends on

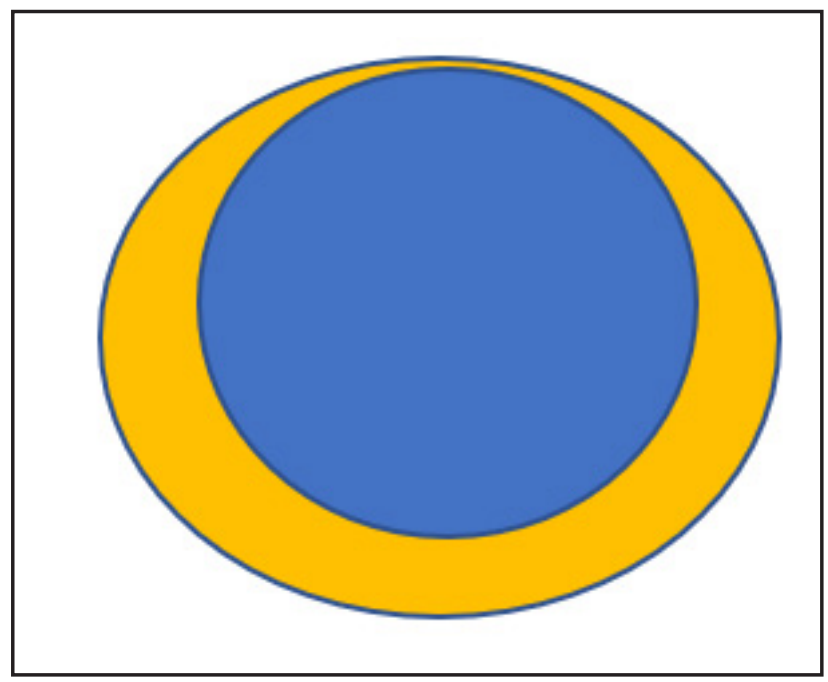

Fig. 11b. Cartilage on pericartilagenous flap

the size of the defect in the tympanic membrane and the status of the ossicular chain including the presence and position of the handle of the malleus. Spongostan may be placed in the middle ear with this graft.

In Fig. $11 \mathrm{a}$ and $\mathrm{b}$ if the blue circle represents the area of the tympanic membrane then the graft shape used for a total, subtotal or large anterior perforation is shown here. Three "outpouches" are created in the temporalis fascia which are then placed on the anterior, inferior and posterior ear canal walls. This appears to successfully support the graft and often no spongostan ${ }^{\mathrm{TM}}$ (absorbable haemostatic gelatin sponge) is required in the middle ear.

The graft positioning: In the underlay technique, a graft to repair the perforation is laid medial to the position of the tympanic annulus. Since stable structures such as the annular ligament and anterior wall of the protympanum are present lateral to the graft, this technique eliminates the possibility of blunting. In the overlay technique the graft is placed lateral to the position of the tympanic annulus, but this technique has the risk of blunting of the anterior angle.

The author uses an underlay technique and after the edge of the perforation is freshened, the tympanomeatal flap is lifted 2 to 10 o'clock like a bucket handle (Fig.12a). An anterior bony canalplasty may be 
required to improve visualisation and access to place the temporalis fascia graft. The graft is then inserted over the incus, under the malleus and once covering the anterior, inferior and posterior ear canal walls the tympanomeatal flap is replaced (Fig.12b).

The overlay technique is also popular although it may be more challenging than the underlay technique if blunting and lateralization of the graft are to be avoided. The graft is placed on the lateral surface of the tympanic membrane after the squamous layer of the remaining tympanic membrane has been carefully removed. A slit in the graft can accommodate the malleus but if the graft is to be placed lateral to the malleus, care must be taken to ensure the malleus is also free of squamous epithelium.

The challenge in myringoplasty is how to secure the grafts anteriorly. One technique "the pocket handkerchief technique" involves making a tunnel in the anterior canal wall and a tag of fascia or perichondrium is then pulled through. It can be a difficult manoeuvre especially when there is a prominent anterior canal wall so an alternative technique using an anterior bony canaloplasty and larger fascia grafts can be employed.

Iain Swan described a technique of making an incision in the tympanic membrane anterior to the perforation through which a tag of fascia or perichondrium passes through and lies on epithelial surface of the tympanic

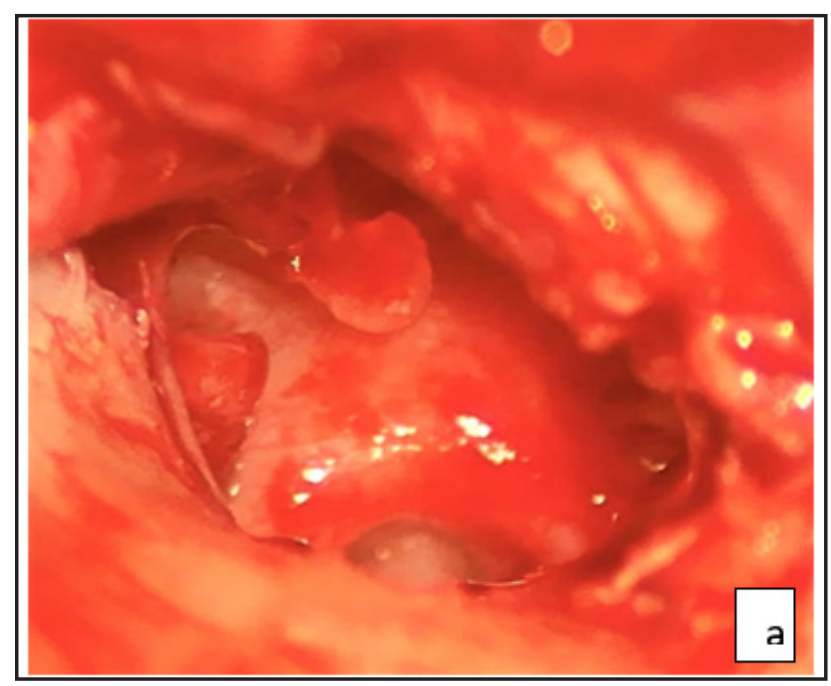

Fig. 12a. Tympanmeatal flap raised 2 to 10 o'clock membrane. Various techniques have been utilised over the years to improve the success rates of myringoplasty for total and subtotal perforations. ${ }^{18}$ Pfleiderer and Moffat in 1987 described a technique utilizing a total tympanic graft of formalized autologous temporalis fascia in 29 patients with total closure of the defect being achieved in $93 \%$ of cases. ${ }^{19}$ The overlay technique is used by some in this situation but again by performing an anterior bony canaloplasty and using a large enough graft that can cover the anterior, inferior and posterior ear canals a successful myringoplasty can be achieved. The endoscopic technique gives great visualisation but can be challenging at times having to manipulate the graft one handedly.

Butterfly grafting is increasing in popularity especially as the endoscopic myringoplasty is being employed7. The cartilage is harvested using a punch biopsy and the rim split so that after the edge of the perforation has been freshened the graft is inserted so a phlange is inserted either side of the tympanic membrane (Fig. 13). This technique enables placement of the graft without raising tympanomeatal flaps.

\section{Surgeon and anaesthetic factors}

Surgery is an apprenticeship and we have all been taught different techniques by our mentors, taking to heart their

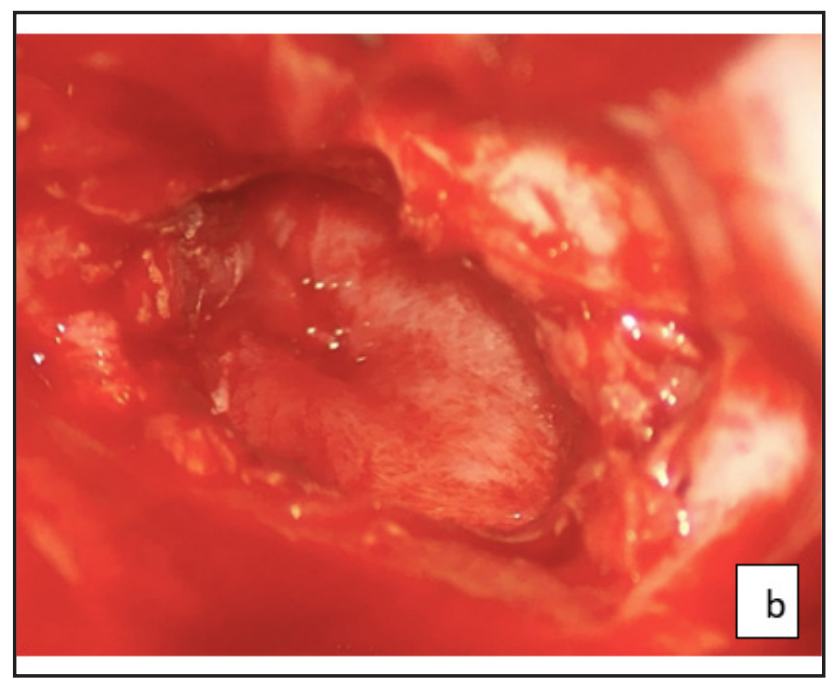

Fig. 12b. Replaced over temporalis fascia 


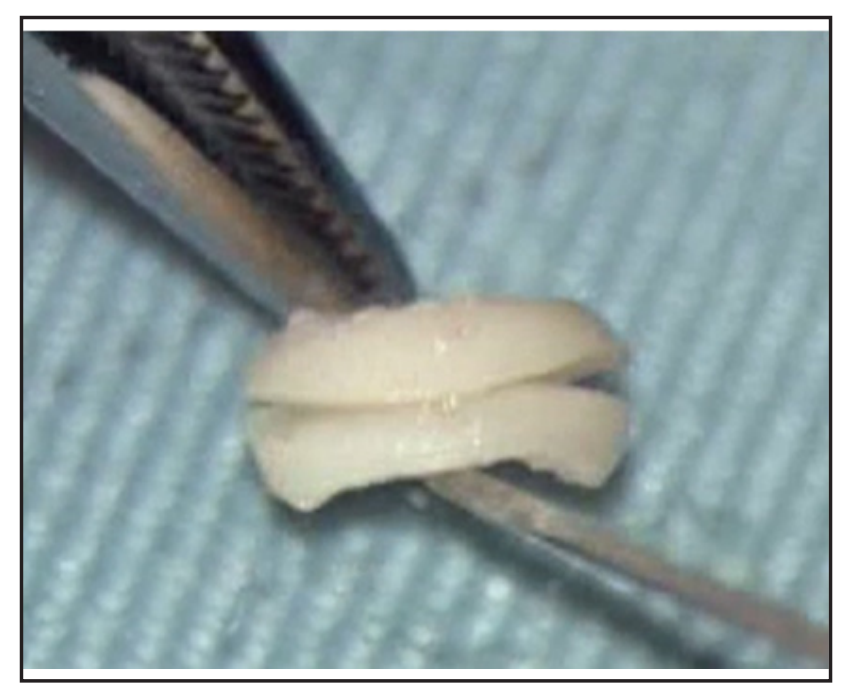

Fig.13. The punch biospy produces a disc which is partially split to form 2 phlanges that lie either side of the tympanic membrane to close the perforation

advice and beliefs depending on our experience at the time and the status of our teacher. We therefore become familiar and comfortable with these techniques. The introduction of endoscopic ear surgery has highlighted other surgeon factors. Interestingly, the handedness of the surgeon can affect the preferred surgical approach. For a right-handed surgeon there is a preference to operate on inferior and posterior perforations in a right ear since the endoscope is held in the left hand out of the way of the instrument hand. The left ear however is preferred by a right-handed surgeon when performing a tympanoplasty to remove cholesteatoma since once again the endoscope is out of the way.

Less experienced surgeons may find permeatal surgery using the microscope challenging but with surgical experience it becomes easier to work in a confined space often operating with only one hand. Many of these skills and those learnt for FESS are transferable to learning endoscopic ear surgery.

The surgeon plays an important contribution to the bloodless field. It was found that when the ear canal was injected with lignocaine and adrenaline, the patient's pulse and blood pressure increased perhaps due to pain. This is prevented by placing putting a cottonoid soaked in lidocaine and adrenaline into the ear canal in the anaesthetic room prior to induction. A small volume, single very slowly administered lidocaine and adrenaline injection in the so called "vascular strip" avoiding blebs is required. The tragus is also injected when cartilage and perichondrium are to be harvested. By removing hair from the ear canal the number of passages of the scope to clean it of blood on passing it down the ear canal is reduced and so this saves operating time in the long run. Care must be taken not to cause trauma to the ear especially the first few times the endoscope and instruments are inserted or removed for each ear before a "memory" of the action is made. Warm saline irrigation can also be useful to reduce bleeding. Patience is required as well as Adrenaline soaked cottonoids.

The key to successful myringoplasty and particularly in Total Endoscopic Ear Surgery (TEES), is a bloodless field and the role of the anaesthetist should not be underestimated. Wormold had shown that TIVA using remifentanil and propofol significantly reduced bleeding during FESS.20 A pulse of 50bmp and a mean arterial pressure of $50 \mathrm{~mm} \mathrm{Hg}$ has been advised. Further vasoconstriction is achieved when the anaesthetist hyperventilates the patient so the expired $\mathrm{PCO} 2$ is reduced to 4 . The use of tranexamic acid also plays a significant role and it should be administered soon after induction since it takes 20 minutes to work. The use of endotracheal tubes makes it easier for the anaesthetist to hyperventilate the patient and this also avoids the placement of a poorly sitting laryngeal mask which can result in venous engorgement.

\section{Equipment availability}

This can influence the surgical approach. Some units have limited resources and by expanding the repertoire of surgical techniques, additional lists may be able to be run concurrently. Permeatal myringoplasty can be performed using the microscope or endoscopically, the microscope is usually used when a postaural approach is necessary.

Fortunately, EES can be set up without the need to purchase new equipment. FESS endoscopes of $4 \mathrm{~mm}$ diameter can be utilised initially. Endoscopes are available in $2.7 \mathrm{~mm}, 3 \mathrm{~mm}$ and $4 \mathrm{~mm}$ diameter. The larger diameter improves the quality of the image obtained however the relative bulk within a narrow 
ear canal can be prohibitory and so a $3 \mathrm{~mm}$ scope is usually recommended. A 0 degree scope is easiest initially especially for straight forward myringoplasties. Standard ear instruments can be used and as well as employing the use of an artery clip to bend fine ends (Fig.14).

\section{The impact of the Covid-19 pandemic}

This pandemic has impacted on the delivery of elective surgery in a number of ways. Hospitals have stopped elective activity, diverting staff and estates to meet the demands of patients with severe symptoms from Covid-19 infections. As activity returns the delivery of elective surgery including myringoplasty is likely to change. Firstly, there is the need to protect patients and staff. It is recognised that patients developing Covid-19 shortly after having a general anaesthetic have a significant mortality (up to $23.8 \%$ ). ${ }^{21}$ Pre-operative isolation of the household and swabbing regimes has been advocated as well as considering using local and avoiding general anaesthesia. It is recognised that the virus Covid-19 can be found in the nasopharynx even in asymptomatic patients and it is known that other coronaviruses can be found in the middle ear mucosa of patients with infection. ${ }^{22}$ Concern has therefore been raised about the risk to surgeons and other members in the operating theatre of exposure to the Covid-19 virus when carrying out surgery in patients with perforated tympanic membranes. A viral plume could potentially occur during diathermy, suction irrigation and highspeed drilling. Full PPE is advocated but presently the use of visors and goggles is difficult when using a traditional microscope. Some centres are assessing the expensive exoscope systems whilst others are forming a "tent" to exclude the surgical field from the rest of the operating theatre. ${ }^{23}$ Other surgeons are adopting the endoscopic surgical approach wherever possible.

\section{Examples of different approaches to myringoplasty}

A permeatal microscopic approach favours posterior perforations as shown in figure 8. This ear is more challenging due to retraction of the tympanic membrane onto the stapes. The endoscopic approach provides

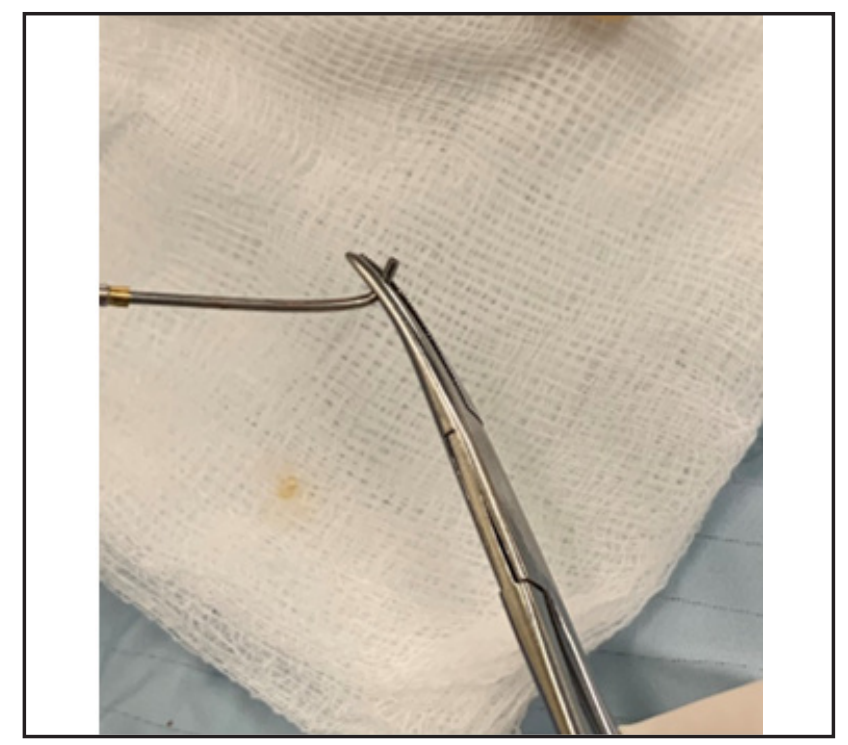

Fig. 14. Using an artery clip to bend a fine end to enable suction around a corner.

better visualisation although depth perception can be difficult when initially using an endoscope. The author found that by using the first canal incision knife with a $3 \mathrm{~mm}$ diameter as a guide, the incision can be made about $6 \mathrm{~mm}$ from the annulus. The tympanomeatal flap should be raised on a broad front using a cottonoid soaked in adrenaline to reduce bleeding, protect the fragile tympanomeatal flap when using suction and as a retractor. When using the endoscopic approach it may be easier to use a smaller graft, often cartilage on a perichondrial flap.

The post aural approach is favoured in young children and in other patients where the ear canal is narrow or if it is tortuous as shown in Fig. 15, particularly if combined with an anterior, subtotal or total perforation.

In revision surgery where tragal cartilage and perichondrium has already been utilised and if Biodesign is not available, a post-aural incision in part to obtain temporalis fascia may be preferred.

If a tympanomeatal flap is raised, the bony meatal wall needs to be covered either with skin or fascia optimizing epithelization and to prevent canal stenosis occurring. It is important to carefully replace the skin to avoid epithelial pearls forming in the ear canal. Many regimes of packing the ear are used varying in the materials being used from gelfoam only to BIPP 


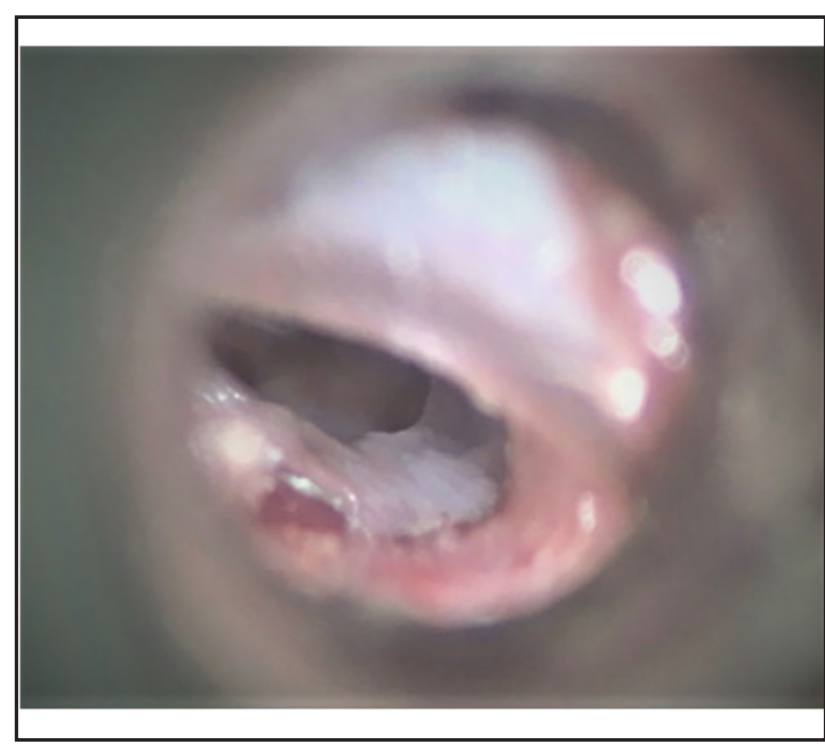

Fig.15. A postauricular approach is easier when there is a tortuous ear canal.

with variation in the length of time the packing is to stay in situ. The author covers the graft with gelfoam soaked in Gentisone HCTM which is then covered with two Betnovate $\mathrm{C}^{\mathrm{TM}}$ ointment-soaked ribbon gauze inserted into the ear canal. All are daycase procedures and if a postaural incision has been used, the head bandage is removed by the patient the day after surgery. The Betnovate $\mathrm{C}$ ribbon gauze is removed at 7 to 10 days. Gentisone HC is used for 4-5 days, the ear is otherwise kept dry for 6 weeks when they are reviewed with a hearing test. If healed no water precautions are required. I then see them at 6 months and at a year with a repeat hearing test on both occasions before discharge from the clinic.

\section{Results of myringoplasty}

The author'sear surgery datahasbeen contemporaneously entered on to the common otology audit. The residual perforation rate is $4.8 \%$ ie over $95 \%$ success rate in over 400 pars tensa perforations. A review of the results of myringoplasty using an endoscopic approach introduced to the author's practice in May 2018, found that in 38 procedures with at least 9 months follow-up, there was a failure of the 4th procedure and another at 6 months post-operatively indicating a $94 \%$ success rate.
Complications: following myringoplasty do occur and myringitis can be more troublesome than a residual perforation. Myringitis is an inflammatory condition of the tympanic membrane and may result from trauma to the tympanic membrane resulting in de-epithelization of the outer surface. Blevins et al in 2001 found 45 ears with chronic (ie more than one month) of myringitis in 750 patients, $60 \%$ had had otological procedures. ${ }^{24}$ Levi et al in 2013 found a history of previous myringotomy or myringoplasty in $80 \%$ of children with this granular myringitis. ${ }^{25}$ This disease is otherwise rare in children. Kim et al in 2011 found it was more common in females. ${ }^{26}$

Blevins reported that topical treatment with antibiotic ear drops was the treatment of choice. ${ }^{24}$ Jung in 2002 described diluted vinegar, ${ }^{27}$ van der Meer in 2010, diluted hydrogen peroxide ${ }^{28}$ although this and silver nitrate may increase the risk of iatrogenic perforations.

Carbon dioxide laser has been used by Jang et al in 2006 and surgical excision and reconstruction with underlay or overlay technique results in a recurrence rate of $1 \%{ }^{29}$

If a residual perforation if present the question arises as to when one should perform revision surgery. In children under 12 years it may be prudent to wait at least a year to see if spontaneous closure occurs. If the graft was successful initially but failed after a further ear infection a review of the frequency of ear infections and whether this is better following the myringoplasty needs to be considered. Prinsley et al in 2017 found the success rates for revision surgery to be like those of primary surgery. ${ }^{30}$

Perhaps it is surprising that only a few patients develop otitis externa following a myringoplasty considering the numbers that occur after ear syringing and the use of cotton buds and other foreign objects placed in the ears. The author's practice of swabbing ears peri-operatively found some correlation of post-operative infection with those with cholesteatoma or peri-operative ear discharge. Myringoplasty on dry perforations do not require perioperative antibiotics unless otherwise indicated by other patient factors.

Otitis externa usually settles quickly after aural toilet (but care must be taken not to micro-suction the 
graft) and topical antibiotic with steroid ear drops. Occasionally infection may result in loss of the graft.

Myringoplasty is not "sold" to improve hearing however hearing can improve especially in posterior, subtotal or total perforations. ${ }^{2}$ Hearing can deteriorate post-operatively if excessive manipulation of the ossicles has taken place or glue ear develops.

Very occasionally small epithelial pearls may form and If small these can be removed in the clinic after local anaesthetic cream has been applied. If cholesteatoma has developed within the middle ear then further preoperative investigations is required prior to removal in the operating theatre.

\section{Conclusion}

A number of factors are taken into consideration often subconsciously, when planning whether and how to perform a myringoplasty. It is useful to have the ability to use a range of techniques, being aware of their limitations and to participate in audit to know personal success rates especially when new techniques are adopted.

\section{References}

1. Rollin M, Rogers P, Robinson P. Natural history of paediatric tympanic membrane perforation. Otol Neurotol. 2011; 32(2):246-51

2. Phillips J, Yung M, Nunney, I. Myringoplasty outcomes in the UK. J Laryngol Otol. 2015; 129(9): 860-4. doi:10.1017/ S002221511500198X

3. Vrabec JT, Deskin RW, Grady JJ. Meta-analysis of pediatric tympanoplasty. Arch Otolaryngol Head Neck Surg. 1999;125(5):530-4. doi:10.1001/archotol.125.5.530

4. Hardman J, Muzaffar J, Nankivell P, Coulson C. Tympanoplasty for chronic tympanic membrane perforation in children: systematic review and meta-analysis. Otol Neurotol. 2015; 36:796-804

5. Raine C, Singh S. (1983). Tympanoplasty in children A review of 114 cases. J Laryngol Otol. 1983; 97(3):217-21. doi:10.1017/ S0022215100094032

6. Grenner J. How I do it: meatoplasty of the ear canal. J Otolaryngol. 1996; 25(3):188-90

7. Rourke T, Snelling J, Aldren C. Cartilage graft butterfly myringoplasty: how we do it. Clinical Otolaryngology 2010; 35(2):135-8
8. Singh GB, Sidhu TS, Sharma A. Tympanoplasty type 1 in children: an evaluative study. Int J Pediatr Otorhinolaryngol. 2005; 69:1071-6. doi: 10.1016/j.ijporl.2005.02.016

9. Lee P, Kelly G, Mills RP. Myringoplasty: does the size of the perforation matter? Clin Otolaryngol Allied Sci. 2002; 27(5):331-4. doi:10.1046/j.1365-2273.2002.00590.x

10. Yung MW. Myringoplasty for subtotal perforation. Clin Otolaryngol. 1995; 20:241-5. doi: 10.1111/j.1365-2273.1995. tb01858.x

11. Mohammed Tawab H, Mahmoud Gharib F, Algarf, T M, ElSharkawy LS. Myringoplasty with and without Cortical Mastoidectomy in Treatment of Non-cholesteatomatous Chronic Otitis Media: A Comparative Study. Clinical medicine insights Ear Nose Throat 2014; 7:19-23. doi: 10.4137/CMENT. S17980

12. Yoon TH, Park S-K, Kim JY, Pae KH, Ahn JH. Tympanoplasty, with or without mastoidectomy, is highly effective for treatment of chronic otitis media in children, Acta Oto-Laryngologica 2007; 127:sup558, 44-8. DOI: 10.1080/03655230701624855

13. Nicholas BD, O'Reilly RC. Is cartilage preferable to fascia myringoplasty in children?. Laryngoscope 2010;120(11):21362137. doi:10.1002/lary.21006

14. Lee DY, Kim YH. Can Fat-plug Myringoplasty Be a Good Alternative to Formal Myringoplasty? A Systematic Review and Meta-analysis. Otol Neurotol. 2018;39(4):403-9. doi:10.1097/ MAO.0000000000001732

15. D’Eredità R. Porcine small intestinal submucosa (SIS) myringoplasty in children: a randomized controlled study. Int J Pediatr Otorhinolaryngol. 2015; 79(7):1085-9

16. James AL. Endoscope or microscope-guided pediatric tympanoplasty? Comparison of grafting technique and outcome. Laryngoscope 2017;127(11):2659-64

17. De Zinis LO, Berlucchi M, Nassif N. Double-handed endoscopic myringoplasty with a holding system in children: preliminary observations. Int J Pediatr Otorhinolaryngol. 2017; 96:127-30

18. Singh M, Rai A, Bandyopadhyay S, Gupta SC. Comparative study of the underlay and overlay techniques of myringoplasty in large and subtotal perforations of the tympanic membrane. J Laryngol Otol. 2003;117(6):444-8. DOI: $10.1258 / 002221503321892262$

19. Pfleiderer AG, Moffat DA. The fascia form graft: a technique for the repair of large perforations of the tympanic membrane. Clin Otolaryngol Allied Sci. 1988;13(6):427-34. doi:10.1111/j.1365-2273.1988.tb00316.x

20. Wormald PJ, van Renen G, Perks J, Jones JA, Langton-Hewer $\mathrm{CD}$. The effect of the total intravenous anesthesia compared with inhalational anesthesia on the surgical field during endoscopic sinus surgery. Am J Rhinol. 2005;19(5):514-20

21. COVID Surg Collaborative. Mortality and pulmonary complications in patients undergoing surgery with perioperative SARS-CoV-2 infection: an international cohort study [published 
online ahead of print, 2020 May 29] [published correction appears in Lancet. 2020 Jun 9;:]. Lancet 2020; 396(10243):2738. doi:10.1016/S0140-6736(20)31182-X

22. Heikkinen T, Thint M, Chonmaitree T. Prevalence of various respiratory viruses in the middle ear during acute otitis media. N Engl J Med. 1999; 340:260-4

23. Hellier W, Mitchell T, Thomas S. Mastoidectomy in the COVID era- the 2 microscope drape method to reduce aerosolization. Br Soc Otology. https://www.entuk.org/sites/ default/files/Mastoidectomy $\% 20 \mathrm{in} \% 20$ the $\% 20$ COVID $\% 20$ Era\%20\%E2\%80\%93\%20The\%202.pdf

24. Blevins NH, Karmody CS. Chronic myringitis: prevalence, presentation, and natural history. Otol Neurotol. 2001; 22(1):310. doi:10.1097/00129492-200101000-00002

25. Levi JR, Ames JA, Gitman L, et al. Clinical characteristics of pediatric granular myringitis. Otolaryngol. Head Neck Surg., 148
(2013), pp. 291-6. https://doi.org/10.1177/0194599812470433

26. Kim YH. Clinical characteristics of granular myringitis treated with castellani solution Eur. Arch. Oto-Rhino-Laryngol.2011; 268:1139-46. https://doi.org/10.1007/s00405-011-1500-7

27. Jung HH, Cho SD, Yoo CK, et al. Vinegar treatment in the management of granular myringitis. J. Laryngol. Otol. 2002; 116:176-80 PMID: 11893257

28. Van der Meer G. Granular myringitis: report of three prospective studies evaluating treatment options. Clin. Otolaryngol. 2010; 35:242-3. https://doi.org/10.1111/j.1749-4486.2010.02118.x

29. Jang C, Kim Y, Cho Y, Wang P. Endoscopy-aided laser therapy for intractable granular myringitis. J Laryngol Otol. 2006; 120(7):553-5. doi:10.1017/S0022215106000739

30. Prinsley P. Results of revision myringoplasty: Are they different to those of primary myringoplasty? J Laryngol Otol.2017; 131(4): 316-8. doi:10.1017/S0022215117000202. 